\title{
NUMERICAL EXPERIMENTS WITH LOW SNR DATA IN RADIO INTERFEROMETRY
}

\author{
P.N. WILKINSON and P. WOODALL \\ University of Manchester, Nuffield Radio Astronomy Laboratories, Jodrell \\ Bank, Macclesfield, Cheshire, SK11 9DL, United Kingdom
}

\begin{abstract}
We outline the results of numerical experiments on low SNR interferometer data. We confirm several theoretical analyses of the effect of averaging on visibility amplitude and closure phase data. However the triple product method for estimating the closure phase, which is useful in optical interferometry, does not provide a significant improvement over existing practice in radio interferometry. We introduce a way of using the triple product to estimate visibility amplitudes from arrays dominated by a single large telescope.
\end{abstract}

\section{INTRODUCTION}

There has been much recent interest in the problem of recovering the phase and amplitude of weak signals from interferometers. In the radio domain the concern is mostly with data obtained at each end of the 'window' i.e. at metre/decametre wavelengths and at millimetre wavelengths. Here the poor phase stability severely limits the allowable coherent integration times, the signal-to-noise ratios (SNRs) may therefore be low $(\leq 2)$ and phase referencing is not possible. In the optical domain these problems are much worse. Selfcalibration techniques, which operate directly on the visibility phases, may not produce a useful image starting from such poor data. One way forward is to average up the good observables, the closure phases, until their SNR is $\geq 2$ and then to apply standard imaging techniques. However, if the the amplitude and phase data are averaged independently the spurious bias on the averaged amplitudes must be corrected. In order to examine the effects of amplitude bias and to see if any of the ideas developed for dealing with optical data could be useful for the analysis of low-frequency MERLIN data, we performed a series of numerical tests with simulated data.

\section{THE TESTS}

Cornwell (1987) published a useful paper in which he clarified the relaiionship between speckle masking (also called triple correlation) techniques, deveioped by optical workers and phase closure methods pioneered by radio astronomers. 
He drew attention to the fact that the closure phase is merely the argument of the triple product, $T_{i j k}$, from a triangle of baselines $i j k$ where

$$
T_{i j k}=A_{i} A_{j} A_{k} e^{i\left(\phi_{i}+\phi_{j}-\phi_{k}\right)}
$$

and gave a numerical demonstration of the fact that, for low SNR data, the best estimate of the closure phase is obtained by averaging successive triple product vectors and then taking the phase part at the end. As the SNR on each baseline baseline approaches unity, simple arithmetic (scalar) averaging of the closure phase produces a biassed estimate which progressively approaches zero phase as the SNR is reduced. The triple product method, however, produces an unbiassed answer even when the individual samples have very low SNR.

We wrote software to calculate the closure phase by various methods, as a function of integration time. As a first test we repeated Cornwell's (1987) calculation and confirmed his Fig. 1 down to an initial SNR of 0.333 per baseline. Scalar averaging is a poor way to estimate the closure phase from many samples - it is not used by the VLBI community (see below). Note, however, that when the individual SNRs are $<1$ the SNR of the closure phase derived from averaging $N$ samples of the triple product, improves much more slowly than $\sqrt{N}$ (Cornwell 1987). In these numerical experiments each final point was the average of 100 realizations of 10000 individual closure phases. This slow increase in SNR restricts the usefulness of triple product methods in radio interferometry, compared with optical interferometry on a single polarmounted telescope. This is because our baselines are continuously rotating relative to the source and one cannot, therefore, integrate for arbitrarily long periods without smoothing the visibility function. Woan and Duffett-Smith (1988) then showed that, for an initial SNR better than $\sim 0.5$, an almost equally good value for the closure phase is obtained by replacing the measured, noisy, amplitudes in the triple product vector by unit amplitude. We confirmed their result numerically.

We also investigated ways of removing the bias which follows a separate averaging of the amplitudes. This is a long-studied problem in interferometry (e.g. Wilkinson 1971; Moran 1976). We tested our software by confirming the calculated Ricean bias on the averaged amplitudes as a function of initial SNR. We then investigated two methods of removing the bias. First we tested the conventional method (see also Rogers et al. 1984) of unbiassing the averaged amplitudes:

$$
A_{e s t}^{2}=\left[1 / N \Sigma A_{b i a s}^{2}\right]-2 \sigma^{2}
$$

where $A_{\text {est }}$ is the estimated true signal amplitude, $\sigma^{2}$ is the variance of the sin or cosine channel and $A_{b i a s}$ is the amplitude of the biassed data. This works satisfactorily but is rather sensitive (being the subtraction of two large numbers to produce a small one) and success is dependent on estimating the 
correct value for $\sigma^{2}$ from the data. Secondly, we investigated a special case of estimating the amplitude using the modulus of the averaged triple product. If an array is dominated by a large telescope, the SNR on the baselines to the dominant telescope will be many times better than those on the other baselines. A triangle of baselines including the dominant telescope may therefore have two baselines which produce good SNR data (amplitudes $A_{i}, A_{j} \geq 3: 1$ ) and one (amplitude $A_{k}$ ) on which the SNR is $\leq 1$. In this special case the modulus of the averaged triple product, $\mid \overline{T_{i j k} \mid}$, can be used to recover the amplitude of the weak fringes using:

$$
\overline{A_{k}}=\left|\overline{T_{i j k}}\right| / \overline{A_{i}} \overline{A_{j}}
$$

If their SNR is $\geq 3: 1, \overline{A_{i}}$ and $\overline{A_{j}}$ are simply obtained from the arithmetic average of the amplitudes on the baselines involving the large telescope. Fig. 1 illustrates the effect of amplitude bias on incoherently averaged data and its removal via equations (2) and (3).
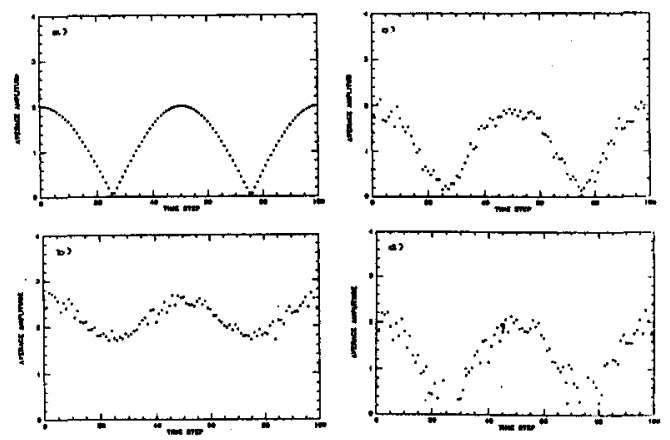

Fig. 1. a) Noise-free amplitude data. b) Arithmetically averaged amplitude data with input $S N R=0.5 ; 100$ input points per output point. c) Averaged data after unbiassing via equation (3). d) Averaged data after unbiassing via equation (2).

We then turned to the CIT VLBI software package. We wrote a version of the AVERAGE program using the triple product method and compared the results with those from the standard version of AVERAGE. For an input SNR of 0.5 on each baseline the differences are small. On examining the code, we discovered that the CIT method of averaging the closure phase involves averaging the sine and cosine components of unit vectors, whose phase angles are the successive closure phases. This is equivalent to triple product averaging using unit amplitudes. As we mentioned earlier, the difference between using the correct amplitudes and unit amplitudes should be small. A version of AVERAGE which uses the triple product is now available. We then found that the UNBIAS program in the CIT package produced results on simulated data which we could not understand. We therefore wrote a new version which implements the standard unbiassing method using equation (2). This produced predictable results. 
Finally we ran a series of imaging tests, with simulated low SNR data from simple double sources, to investigate the various ways of treating averaged closure phases and amplitudes. We adopted the following simple strategy for imaging: i) Average the closure phase data using the triple product method, or the standard CIT method, for as long as possible whilst still preserving any 'structure' seen in the amplitudes. ii) Unbias the incoherently averaged data, using equation (2), having carefully established the value of $\sigma$ for the sin and cosine channels. iii) Reconstruct estimated visibility phases from the averaged closure phases using the original Readhead and Wilkinson (1978) method (the CIT program PHASE). iv) Make a hybrid map as usual using AMPHI, INVERT and CLEAN (e.g. Wilkinson, 1989).

Briefly the results were as follows: unless the SNR of the averaged closure phase is $\geq 2$ on most triangles then our hybrid mapping strategy often failed. It is possible to see the advantage of triple product averaging of closure phase (cf. the standard CIT method) in a hybrid map but the effect is small. Unbiassing the incoherently averaged amplitude data is more important than the specific method used to average the closure phases. The effect of the amplitude bias is to add a 'd.c. offset' to each baseline - crudely this can mimic the effect of a point source at the centre of the map.

Overall then, we didn't find out much that was new. Nevertheless we confirmed various theoretical analyses of the effect of averaging on amplitude and phase data, and we found that for practical purposes the triple product offers little advantage in low SNR radio interferometry. Averaging up the data enough to take advantage of the optimum estimator of closure phase, almost certainly results in excessive smoothing of the visibility function of any but the simplest sources.

This work formed part of P. Woodall's M.Sc. thesis. More details of our tests, including the analysis of real MERLIN data on the gravitational lens MG1131+0456, are given in the thesis. Copies are available on request.

\section{REFERENCES}

Cornwell, T. J. 1987, Astr. Ap., 180, 269.

Moran, J. M. 1976, in Methods of Experimental Physics, ed M. L. Meeks, Vol, 12 part C, Academic Press (New York), p. 228.

Readhead, A. C. S. and Wilkinson, P. N. 1978, Ap. J., , 223, 25.

Rogers, A. E. E., Moffett, A. T., Backer, D. C. and Moran, J. M. 1984, Radio Sci., $19,1552$.

Wilkinson, P. N. 1971, Ph.D. Thesis, Univesity of Manchester.

Wilkinson, P. N. 1989, in Very Long Baseline Interferometry, Techniques and Applications, ed M. Felli and R. E. Spencer, NATO ASI Series, Vol. 283, p. 69.

Woan, G. and Duffett-Smith, P. J. 1988, Astr. Ap., , 198, 375 